\title{
DYNAMICS OF ADIABATIC SHEAR
}

\author{
D.E. GRADY \\ Organizarion 1543, Sandia National Laboratories, \\ Albuquerque, New Mexico 87185, U.S.A.
}

\begin{abstract}
The formation of adiabatic shear bands is examined with an approximate analytic model. The shear band is viewed as a propagating feature with a well-defined front. The shear band is further partition into a shear-band process zone within which most of the adiabatic heating and shear stress relaxation occurs, followed by a quasisteady zone within which little dissipation occurs. Although a one-dimensional analysis of the shear-band dynamics is initially pursued, the analysis is then used to calculate properties of the inherently two-dimensional shearband process zone. The length and width of the process zone is calculated along with the shear displacement. The model is further used to calculate the energy dissipation within the shear-band process zone. The flow field within the vicinity of the process zone is also examined. Calculated properties of the shear-band process zone are compared with available experimental data.
\end{abstract}

\section{1.- Introduction}

The formation of adiabatic shear bands in metal structures subjected to rapid shearing through explosive or impact loading is an important deformation mechanism. The characteristics of shear banding have been investigated extensively over the several past decades. Metallurgical studies have been effective in revealing the morphology of the shear-banding processes and have pointed toward possible deformation mechanisms $\left({ }^{1,2,3}\right)$. The combination of careful metallurgical investigation along with controlled dynamic loading instrumentation and associated diagnostics has lead to further improvements in understanding of the stress and strain dynamics governing shear-band formation and evolution $\left({ }^{4}, 5,6\right)$. Recent investigations with time-resolved photographic and infrared radiation diagnostics have provided further revealing data on the dynamic thermoplastic processes of shear banding $(7,8)$. These data collectively provide necessary bench marks for testing the extensive modelling and analytic efforts which have been pursued to explain various aspects of adiabatic shear-banding phenomena $(9,10,11,12,13,14,15,16)$.

The goals of the present study have focused on achieving a better understanding of energy dissipation in the adiabatic shear-banding process. The recent study of Marchand and Duffy $\left(^{8}\right)$ indicates that a shear band does not simultaneously evolve along the full plane of shear. Rather, their data suggests that shear bands originate within a localized region and have well defined fronts which propagate with finite velocities along the plane of shear deformation much like mode II cracks. Thus, in this work, we pursue the idea of a propagation shear band with a shearband-tip process zone within which the preponderance of energy dissipation takes place.

While recognizing the two-dimensional nature of a shear-band process zone, a one-dimensional model of shearband formation is nevertheless initially pursued and applied to the two-dimensional phenomena. The present model of adiabatic shear-band evolution involves simplifying assumptions which are intended to render the analysis tractable, and also to lend insight into the physics of the phenomenon. The shear band is modelled as having a fixed thickness. Energy minimum concepts are used to establish a preferred shear-band thickness. Material exterior to, 
but in the vicinity of, the shear band is modelled as rigid-plastic. Material within the shear band is modelled as thermoplastic. A solution method pursued earlier $\left({ }^{17}\right)$ is built upon to complete the analysis.

The analysis is used to calculate properties of the shear-band process zone including the width and length of the process zone, and the shear displacement accumulated within this region. The energy dissipated within the shearband process zone is also calculated from the model. The deformation within the neighborhood of the two-dimensional shear-band process zone predicted from the model is also examined. The calculated results are compared with experimental observations of shear bands.

\section{2.- The shear-band process zone.}

It is reasonable to believe that the evolution of adiabatic shear localization in a rapidly deforming metal involves the propagation of an extending shear band as is suggested by the study of Marchand and Duffy $\left(^{8}\right)$. There will be a shear-band tip or process zone. Within this shear-band process zone, shear stress will be expected to relax from the ambient temperature flow stress value in the metal near the front of the process zone to the significantly lower value at the back of the process zone corresponding to the near-adiabatically heated shear-band material. The thermophysical state within the process zone will be transitional in that stresses and temperatures will change rapidly. Behind the shear band a quasi-steady state is achieved in which stresses and temperatures change slowly. It can also be expected that most of the dissipation in the propagating shear band will occur within this process zone.

In the present analysis the simplifying approximations illustrated in Fig. 1 are made. Namely, it is assumed that shear stress and dissipation rate are finite only within the shear-band process zone. Stress and dissipation rate are assumed zero behind the process zone in the region of quasi-steady behavior. That this behavior is not strictly correct is seen by recognizing that maintaining an elevated temperature within the quasi-steady zone requires a reduced but finite dissipation rate. Nevertheless this approximation lends itself to useful analysis of the shear-band processes.

Three characteristic lengths emerge from the analysis as descriptive of the shear-band process zone. First, is the length of the shear-band process zone $x_{s b p z}$ which, as illustrated in Fig. 1, defines the extent of the shear band through the transition region from inception to entrance into the quasi-steady zone. This dimension is analogous to the process zone length identified in the propagation of a ductile crack. Second, is the characteristic width of the shear-band process zone $x_{s b t w}$. One could expect, in application, that this dimension would be time dependent. The width $x_{s b t w}$ is assumed to be constant in the present model. The third characteristic length is the shear-band tip displacement $x_{s b t d}$ which determines the extent of shear displacement of adjacent walls of the band upon transition into the quasi-steady zone as shown in Fig. 1 . This property would be analogous to crack opening displacement in ductile fracture.
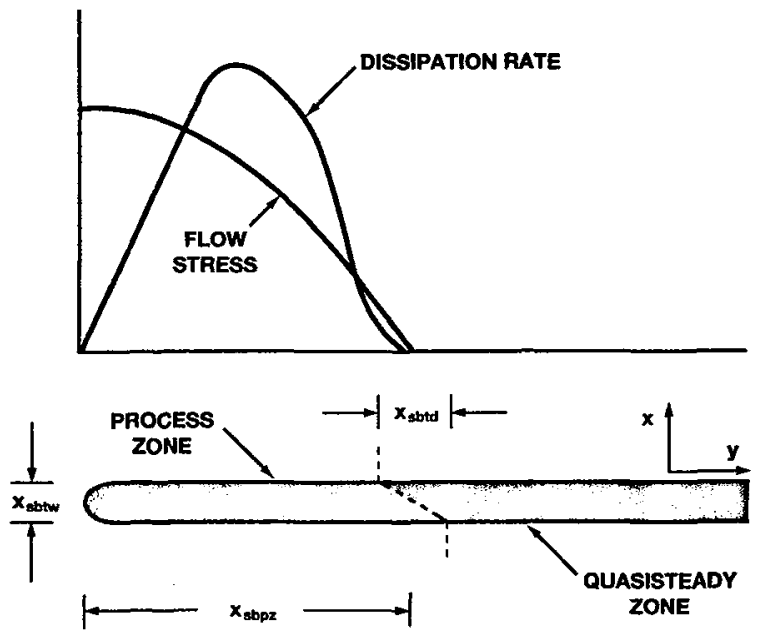

Fig. 1.- Geometry of the front of a propagating shear band. 
In determining these lengths and other properties descriptive of the shear-band process zone in the following analysis, it will be assumed that the goverming thermomechanical differential equations are functions of only a single spacial coordinate $x$ which is the normal distance from the planar shear band as seen in Fig. 1 . The assumed independence of the coordinate $y$ would seem reasonable justified if $x_{s b p z}$ " $x_{s b t d} \gg x_{s b t w}$. The extent to which these conditions are realized in application must be considered.

\section{3.- Analysis of the adiabatic shear-band process.}

The equations goveming planar rigid thermoplastic shear follow from momentum and energy balance. They are,

$$
\begin{gathered}
\rho \frac{\partial v_{y}}{\partial t}=\frac{\partial \tau}{\partial x}, \\
\frac{\partial \theta}{\partial t}=\frac{1}{\rho c} \tau \frac{\partial v_{y}}{\partial x}+\frac{\partial^{2} \theta}{\partial x^{2}},
\end{gathered}
$$

where $v_{y}(x, t)$ is the velocity, $\tau(x, t)$ is the shear stress, and $\theta(x, t)$ is the temperature excursion above ambient in the region of the shear band. When plastic dissipation (assumed to be totally thermal in the present analysis) leads to thermal softening, localization of shear deformation is predicted. Extent of the transient localized region is determined through the governing equations from a competition of thermal diffusion and momentum diffusion effects. In the present study a simpler problem is investigated. The decreased complexity of the material response model is offset by the increase in tractability of the resulting governing equations.

In the model, material within the shear band is considered to be separated from exterior material by a distinct interface and is treated differently. The model has been described in an earlier study $\left({ }^{17}\right)$ and is extended to address the different emphasis of the present investigation. Material response in the vicinity of the planar adiabatic shear band assumed in the model is illustrated in Fig. 2. The sense of the volume-preserving shear flow is indicated in the Figure. The shear band is assumed to have a non-changing thickness $a$. Shear deformation within the band is uniform. Shear flow in the body that is not perturbed by the shear banding process occurs at a constant flow stress $\tau_{y}$. As stress relaxes within the shear band while deformation evolves, stress release propagates outward into the undisturbed media as an interface separating plastic flowing material in front of the interface and rigid material behind.

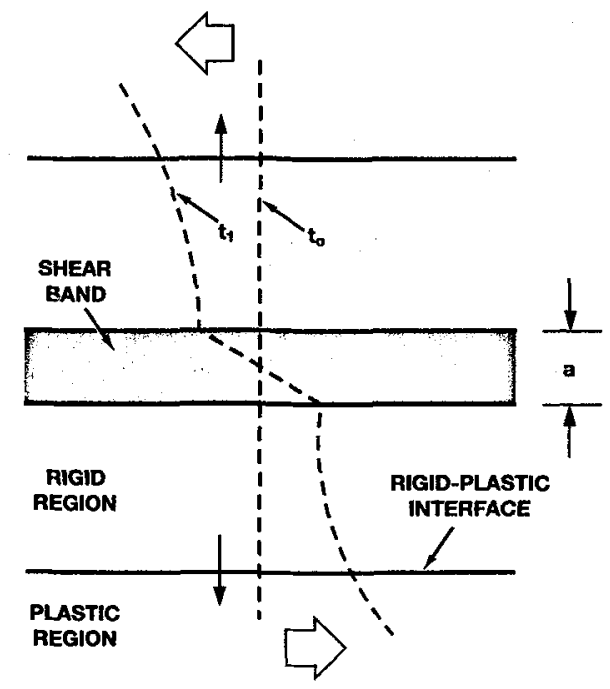

Fig. 2.- Configuration for a rigid-plastic solution for stress release from a one-dimensional thermal softening shear band. 
During deformation, temperature rises within the shear band according to a rule relating heating and plastic dissipation. Heat conduction out of the shear band and into the neighboring media is also accounted for. A heat transfer coefficient relating the temperature difference and the thermal conduction characteristics of the material is defined. Material outside of the shear band is assumed to remain at the ambient temperature. Thus a single temperature $\theta$ characterizes heating above ambient within the shear band.

A Lagrangian coordinate system (coordinate $x$ normal to the shear band and coordinate $y$ in the direction of flow) is attached to the shear band system. The point $x=0$ corresponds to the center of the shear band. It is assumed that the extent of the problem is such that details of the shear band can be collapsed to a boundary condition at $x=0$. A boundary displacement $\psi$ represents the magnitude of shear-band slip at $x=0$. An interface position $\xi$ identifies the Lagrangian position of the rigid-plastic interface.

The solution for the motion follows from a method initially pursued by Mott $\left({ }^{18}\right)$ and extended in later work $\left({ }^{17,19}\right)$. Accordingly, the domain of interest includes the half space $x \geq 0$, which, at $t=0$, is undergoing uniform shear in the $y$ direction. Material response is assumed rigid plastic with the body deforming at a constant strain rate $\dot{\gamma}$ and at a flow stress $\tau_{y}$. Consequently, the initial velocity field is given by $v_{y}(x)=\dot{\gamma} x$. At $t=0$ a shear band initiates in the plane $x=0$ and subsequent stress $\tau$ on this boundary will decrease with time according to the thermoplastic properties of the shear-band material.

Using momentum conservation, energy conservation and compatibility, the system of ordinary differential equations is obtained,

$$
\begin{gathered}
\rho \dot{\gamma} \frac{d \xi}{d t}=\tau_{y}-\tau(\psi, \dot{\psi}, \theta), \\
\frac{d \psi}{d t}=\dot{\gamma} \xi, \\
\frac{d \theta}{d t}=\frac{1}{c_{a}} \tau \frac{d \psi}{d t}-\chi_{a} \theta .
\end{gathered}
$$

In the energy equation $c_{a}$ and $\chi_{a}$ are an area heat capacity and a heat transfer coefficient for the shear band, respectively. These properties are approximated by $c_{a}=\rho c a / 2$ and $\chi_{a}=2 \chi / a^{2}$ as was done in Grady and Kipp $\left({ }^{17}\right) . c$ and $\chi$ are the bulk specific heat and thermal diffusion coefficients, respectively, for the material of interest.

The treatment of equations (3) through (5) will use methods which, although approximate, provide the specific relations for the shear-band process zone which are sought. The approach assumes that the stress difference in equation (3) grows linearly with displacement to the flow stress $\tau_{y}$ at a critical displacement $\psi_{c}$. That is,

$$
\tau-\tau_{y}=\tau_{y} \frac{\psi}{\Psi_{c}}, \quad\left(0 \leq \psi \leq \Psi_{c}\right) .
$$

It is important to recognize that this relation is not a constitutive assumption. It is an assumed form for the solution which simply requires that softening within the shear band lead to this particular stress-displacement behavior. Displacements larger than $\psi_{c}$ are not of interest in the present analysis.

Substitution of equation (6) into equation (3) decouples the mechanical system of equations,

$$
\begin{gathered}
\rho \dot{\gamma} \frac{d \xi}{d t}=\frac{\tau_{y}}{\psi_{c}} \psi, \\
\frac{d \psi}{d t}=\dot{\gamma} \xi,
\end{gathered}
$$


from the energy equation and thermal considerations which are then treated separately. Subject to the initial conditions $\psi(0)=\xi(0)=0$, solutions for equations (7) and (8) are readily found,

$$
\begin{gathered}
\psi=\frac{\tau_{y} \dot{\gamma}}{18 \rho \psi_{c}} t^{3}, \\
\xi=\frac{\tau_{y}}{6 \rho \psi_{c}} t^{2} .
\end{gathered}
$$

From equation (9) a critical time $t_{c}$, when $\psi=\psi_{c}$, is solved for,

$$
t_{c}=\left(\frac{18 \rho \psi_{c}^{2}}{\tau_{y} \dot{\gamma}}\right)^{\frac{1}{3}} \text {. }
$$

Thus solution of the mechanical system of equations leads to a correspondence between a critical displacement of a shear band at which the shear stress relaxes to zero and the time to achieve that displacement. A requirement of energy consistency is now placed on the motion which leads to a further independent correspondence between $t_{c}$ and $\psi_{c}$.

Relaxation of stress during deformation of the shear band is assumcd to be due to the thermal softening nature of the shear-band material. The elementary constitutive relation,

$$
\tau=\tau_{y}(1-\alpha \theta),
$$

where $\alpha$ is a thermal softening coefficient, is assumed to characterize the thermal softening properties of the shearband material. The time dependence of the shear-band temperature resulting from the mechanical solution is then determined,

$$
\theta=\frac{\tau \dot{\gamma}}{18 \alpha \rho \psi_{c}^{2}} t^{3}
$$

It is readily seen by direct substitution that equation (13) does not satisfy the energy equation. Instead, the weaker requirement of global energy consistency is imposed by integrating the energy equation over the critical time $t_{c}$,

$$
\int_{0}^{\theta_{c}} d \theta=\frac{1}{c_{a}} \int_{0}^{t_{c}} \tau \dot{\gamma} d t-\chi_{a} \int_{0}^{t_{c}} \theta d t .
$$

Carrying out the integration leads to,

$$
\psi_{c}=\frac{2 c_{a}}{\tau_{y} \alpha}\left(1+\frac{\chi_{a} t_{c}}{4}\right) .
$$

Equation (11) and (15) provide two algebraic relations which can be solved for the critical displacement $\psi_{c}$ and the critical time $t_{c}$. In particular, solving for the critical displacement yields the implicit relation,

$$
\Psi_{c}^{2}=\frac{32 \tau_{y} \dot{\gamma}}{9 \rho \chi_{a}^{3}}\left(\frac{\alpha \tau_{y}}{2 c_{a}} \Psi_{c}-1\right)^{3} .
$$

At this point, properties specific to the shear band are introduced. Energy dissipated within the shear band as deformation proceeds to a critical displacement $\psi_{c}$ is identified as $\Gamma_{c}=\left(\tau_{y} \psi_{c}\right) / 2$. Consistent with concepts of frac- 
ture mechanics, $\Gamma_{f}$ is an energy per unit area dissipated within one half of the shear band. Also bulk thermal properties for the shear-band material are introduced through the approximations $c_{a}=\rho c a / 2$ and $\chi_{a}=2 \chi / a^{2}$. Substituting into equation (16) yields,

$$
\Gamma_{c}^{2}=\frac{\dot{\gamma} \tau_{y}^{3} a^{6}}{9 \rho \chi^{3}}\left(\frac{2 \alpha}{\rho c a} \Gamma_{c}-1\right)^{3} .
$$

Equation (17) provides an implicit relation for energy dissipated within a shear band as the shear-band stress relaxes from the flow stress $\tau_{y}$ to zero due to adiabatic heating within the band. The dissipation energy is a function of thermomechanical material properties and the width of the shear band. The functional dependence of the dissipation energy on the shear band width is shown in Fig. 3. A local minimum, with increasing dissipation for both larger and smaller shear-band widths, is indicated. The physical processes responsible for the observed behavior are reasonably clear. For thinner bands the enhanced thermal diffusion limits the rate of thermal softening and leads to excessive dissipation. Thicker bands are effectively adiabatic. However, the diffusion of momentum (inertia) into the shear-band vicinity again limits the rate of thermal softening and also leads to excessive dissipation. Shearband thicknesses near the local minimum properly balance thermal and momentum diffusion providing the minimum possible shear-band dissipation.

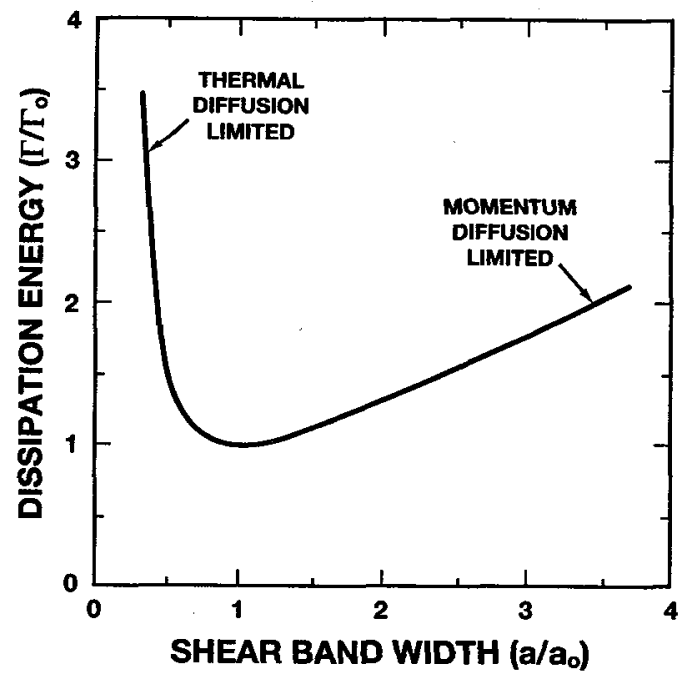

Fig. 3.- Plot of the energy dissipated in the shear-band process zone as a function of the shear-band width.

In the present study it is postulated that in application, forces are brought about which lead to shear-band thicknesses consistent with a minimum dissipation energy. Using equation (17) an optimum shear-band thickness is derived,

$$
a_{o}=\left(\frac{9 \rho^{3} c^{2} \chi^{3}}{\tau_{y}^{3} \alpha^{2} \dot{\gamma}}\right)^{\frac{1}{4}}
$$

The dissipation energy corresponding to a thickness $a_{o}$ is,

$$
\Gamma_{c o}=\frac{\rho c}{\alpha}\left(\frac{9 \rho^{3} c^{2} \chi^{3}}{\tau_{y}^{3} \alpha^{2} \dot{\gamma}}\right)^{\frac{1}{4}}
$$




\section{4.- Calculation of shear-band properties.}

In this Section the one-dimensional rigid-plastic analysis of shear-band evolution will be used to infer the approximate geometry and kinematic properties of the inherently two-dimensional process zone region of a propagating shear band. To quantify the calculation and to provide a focus for discussion, material properties relevant to shear localization in 4140 steel will be used. The same material was used in explosive fragmentation experiments by Grady and Hightower $\left({ }^{20}\right)$, which provided the motivation for the present investigation. Critical material properties are contained therein.

Referring again to the characteristic dimensions of the shear-band process zone identified earlier in Fig. 1, it is concluded that a reasonable estimate of the shear-band width $x_{\text {sbtw }}$ is provided by the optimum shear-band thickness from equation (18),

Based on the material properties for 4140 steel a value of $x_{s b t w}=6.7 \mu \mathrm{m}$ is calculated. A strain rate of $\dot{\gamma}=1.6 \times 10^{4} / \mathrm{s}$ used in equation (18) is consistent with explosive fracture experiments on the same steel (20). This calculated value agrees remarkably well with the thickness of arrested shear bands observed in micrographs of fragments examined in the same work which were about 7 to $10 \mu \mathrm{m}$ in thickness. Studies of Giovanola ( $)$ and Marchand and Duffy $\left({ }^{8}\right)$ on 4340 steel and HY-100 steel, respectively, both yield shear-band thicknesses of about $20 \mu \mathrm{m}$ in torsional Kolsky bar specimens.

Within the present model, energy dissipated in the shear band is assumed to occur entirely within the shear-band process zone. The results are, of course, subject to the approximations introduced there, but should provide a reasonable indication of actual behavior. The analysis leading to equation 19 for the dissipation in the process zone provides the shear-band dissipation energy, $\Gamma_{s}=\Gamma_{c o}$. Again using properties for 4140 steel, a shear-band dissipation energy of $\Gamma_{s}=31,600 \mathrm{~J} / \mathrm{m}^{2}$ is calculated. (A value of $19,000 \mathrm{~J} / \mathrm{m}^{2}$ calculated in the work of Grady and Hightower $\left({ }^{20}\right)$ is in error because $Y$ rather the $\tau_{y}$ was used in equation 19.) Note that $\Gamma_{s}=\rho(c / \alpha) x_{s b t w}$ and, since $\theta_{c}=1 / \alpha$ is the temperature rise through the shear-band process zone, $\Gamma_{s}$ is equal to twice the heat energy developed in the shear band. This leads to the reasonable conclusion that half of the dissipation contributes to temperature rise while half is conducted as heat out of the shear band.

The extent of shear displacement within the process zone $x_{s b t d}$ is readily calculated from

$$
x_{s b t d}=2 \psi_{c o}=\frac{4 \Gamma_{s}}{\tau_{y}}=\frac{4 \rho c}{\alpha \tau_{y}} x_{s b t w} .
$$

For 4140 steel we calculate $x_{\text {sbtd }}=230 \mu \mathrm{m}$. This implies a total shear strain of about 35 within the shear-band process zone. Note that $x_{s b t d} 》 x_{s b t w}$ which was one of the earlier criteria suggested for applicability of the onedimensional rigid-plastic analysis within the process zone.

At this point it is of interest to compare predictions with adiabatic shear-band properties of Giovanola ( ) on 4340 steel. Using high speed photography in conjunction with standard Kolsky bar diagnostics, Giovanola was able to establish the nominal shear-band width along with the shear strain and energy dissipation in the shear band corresponding to the time of loss of shear stress in the adiabatic shear-band process. The properties from this experimental study can be compared directly with the predictions of the present investigation. A measured dissipation energy of $\Gamma_{s}=90 \mathrm{~kJ}$ compares with a prediction of $\Gamma_{s}=44.7 \mathrm{~kJ}$. A measured shear band width of $x_{s b t w}=20 \mu \mathrm{m}$ compares with a prediction of $x_{s b t w}=9.5 \mu \mathrm{m}$. A measured shear-band strain of $\gamma_{s b}=17$ compares with a prediction of $\gamma_{s b}=35$. A shear strain rate of $4000 / \mathrm{s}$ was estimated from Fig. 2 of Giovanola (). The agreement between experiment and theory within a factor of 2 to 3 is encouraging considering the clear differences in conditions and materials, and the simplifying assumptions in the model.

\section{5.- Closure.}

In the present study an analysis of the adiabatic shear-band process in metals based on a one-dimensional rigidplastic model originally pursued by Grady and Kipp $\left({ }^{17}\right)$ has been extended to examine the physical characteristics of the tip of a propagating shear band. Because of the simplicity of the model, physical insight is revealed which is 
not readily extracted from more complex analyses. Application of the model and solutions of the resulting governing equations requires approximating assumptions which may be overly restrictive. On the other hand, results of the analysis seem intuitively reasonable and are in acceptable accord with observed characteristics of dynamic shear bands in steel.

\section{6.- Acknowledgments.}

The author wishes to acknowledge numerous fruitful conversations with Marlin Kipp on the present topic.

\section{7.- References}

/1/ Rogers, H. C., Ann. Rev. Mat. Sci., 9 (1979) 283.

12/ Stelly, M. and Dormeval, R., Met. Appl. of Shock-Wave and High-Strain-Rate Phenomena (edited by Murr, L. E., Staudhammer, and K. P., Meyers, M. A.) Marcel Dekker, New York, (1986) 607.

13/ Timothy, S. P., Acta Metall., 35 (1987) 301.

14/ Costin, L. S., Crisman, E. E., Hawley, R. H. and Duffy, J. 2nd Conf. on the Mechanical Properties of Materials at High Rates of Strain (edited by Harding, J.), The Inst. of Physics, London, (1979) 90.

15/ Dormeval, R. and Ansart, J. P., International Conference on Mechanical and Physical Behavior of Materials under Dynamic Loading, le Editions de Physique, France, (1985) 299.

16/ Pintat, T., Meyer, L. W. and Schrader, H., International Conference on Mechanical and Physical Behavior of Materials under Dynamic Loading, le Editions de Physique, France, (suppl.), (1985) C5-.

7/ Giovanola, J., Impact Loading and Dynamic Behavior of Materials, Vol. 2 (edited by Chiem, C. Y., Kunze, H. D. and Meyer, L. W.) Verlag, Germany, (1987) 705.

/8/ Marchand, A. and Duffy, J., J. Mech. Phys. Solids 36, (1988) 251.

19/ Culver, R. S., Metallurgical Effects of High Strain Rates (edited by Rohde, R. W., Butcher, B. M., Holland, J. R. and Kames, C. H.), Plenum, New York, (1973) 519.

/10/ Bai, Y. L., J. Mech. Phys. Solids 30, (1982) 195.

/11/ Merzer, A. M., J. Mech. Phys. Solids 30, (1982) 323.

/12/ Molinari, A. and Clifton, R. J., J. Appl. Mech. 54, (1987) 806.

/13/ Burns, T. J., J. Appl. Math. 43, (1985) 65.

/14/ Fressengeas, C. and Molinari, A., 1987, J. Mech. Phys. Solids 35,185.

/15/ Coleman, B. D. and Newman, D. C., Acta Mechanica 78, (1989) 263.

/16/ Wright, T. W., J. Mech. Phys. Solids 38, (1990) 515.

/17/ Grady, D. E. and Kipp, M. E., J. Mech. Phys. Solids 35, (1987) 95.

/18/ Mott, N. F., Proc. R. Soc. A189, (1947) 300.

/19/ Kipp, M. E. and Grady, D. E., J. Mech. Phys. Solids 33, (1985) 399.

/20/ Grady, D. E. and Hightower, M. M., Proceedings of the International Conference on Shock-Wave and High-Strain-Rate Phenomena in Materials, San Diego California, (to appear), (1990). 